\title{
Minimally invasive injection laryngoplasty in the management of unilateral vocal cord paralysis after video-assisted mediastinal lymph adenectomy
}

\author{
H. Volkan Kara ${ }^{1}$, Aysegul Batioglu Karaaltin ${ }^{2}$, Ezel Ersen ${ }^{1}$, Elvin Alaskarov², Burcu Kilic ${ }^{1}$, Akif Turna $^{1}$ \\ ${ }^{1}$ Department of Thoracic Surgery, Cerrahpasa Medical Faculty, Istanbul University, Istanbul, Turkey \\ ${ }^{2}$ Department of Ear Nose Throat, Cerrahpasa Medical Faculty, Istanbul University, Istanbul, Turkey
}

Videosurgery Miniinv 2018; 13 (3): 388-393

DOI: https://doi.org/10.5114/wiitm.2018.75886

\begin{abstract}
Introduction: Video-assisted mediastinal lymphadenectomy (VAMLA) is a valuable tool for invasive staging of the mediastinum. Unilateral vocal cord paralysis (UVCP) may occur in patients following VAMLA and may result in secretion retention within the lungs, atelectasis and associated infectious situations such as pneumonia. Minimally invasive injection laryngoplasty (ILP) is the treatment of choice in UVCP.

Aim: To evaluate the efficacy and success of acute minimally invasive injection laryngoplasty for patients with UVCP following VAMLA.

Material and methods: Patients with the symptom of dysphonia following VAMLA were reviewed. All of the patients had UVCP according to the video laryngoscopy examination and had symptoms of aspiration and ineffective coughing. The Voice Handicap Index (VHI) questionnaire and maximum phonation time (MPT) were measured. Minimally invasive ILP was performed under general anesthesia with $1 \mathrm{~cm}$ of hyaluronic acid.

Results: There were 525 consecutive non-small cell lung cancer (NSCLC) patients who underwent VAMLA. Five (0.95\%) of the patients had UVCP and were suffering from aspiration during oral intake and ineffective coughing reflex. Maximum phonation time (MFT) was measured before and after ILP, and the results were $7.1 \pm 1.6$ and $11.1 \pm 2.3$ s, respectively $(p<001)$. The Voice Handicap Index-10 (VHI-10) score was $30.4 \pm 4.7$ and $13.4 \pm 3.5$ $(p<0.01)$, respectively. Patients underwent surgical lung resection. There was no morbidity or mortality.

Conclusions: Unilateral vocal cord paralysis may occur as a complication of VAMLA. ILP may be an active tool for treating UVCP before anatomical lung resection to avoid potential morbidities. Successful management of this complication with multidisciplinary team work may encourage the use of VAMLA more frequently.
\end{abstract}

Key words: minimally invasive injection laryngoplasty, unilateral vocal cord paralysis, video-assisted mediastinal lymph adenectomy.

\section{Introduction}

Accurate TNM staging provides the most reliable information about the prognosis of non-small cell lung cancer (NSCLC) patients [1-3]. Mediastinal lymph node involvement of a tumor is one of the very important limitations for surgical resec- tion so mediastinum should be well evaluated $[1,4]$. Mediastinoscopy has been accepted as the gold standard method for preoperative mediastinal evaluation. Conventional mediastinoscopy has been used commonly, but it has a false negativity rate up to $10 \%$, which is nearly equal to endoso- 
nographic or transbronchial needle aspiration [5] This rate indicates those patients who are not suitable for surgery with the existing evidence-based data but undergo surgery unnecessarily. To improve the efficacy for mediastinal evaluation $\mathrm{Heu}$ rtgen et al. developed the technique of radical video-assisted mediastinoscopic lymphadenectomy (VAMLA) [6]. The primary advantage of VAMLA over conventional mediastinoscopy or videomediastinoscopy is that it reduces the false-negative rate by excising the whole lymphoid tissue with the adjacent fatty tissue $[1,4,6]$. In VAMLA subcarinal, upper and lower paratracheal areas and existing lymph nodes are excised nearly totally [4]. This extended resection may cause damage of the recurrent laryngeal nerve (RLN) mostly on the left side where the nerve lies adjacent to the trachea (Photo 1). Most unilateral vocal cord paralysis (UVCP) cases occur immediately after VAMLA and are suspected by breathy voice and dysphonia. Also patients with UVCP may have dysphagia, ineffective coughing, and fluid aspiration [7, 8].

Unilateral vocal cord paralysis mostly results from injury to the ipsilateral RLN. The injury of the nerve (RLN) may arise from intentional movements during surgical interventions while resecting adjacent structures and transecting the nerve itself [7]. latrogenic anatomical injury of some nerve fibers may produce a transient neurapraxia (due to edema around the nerve) or permanent dysfunction caused by the surgeon's intention to achieve a complete resection of the lymph nodes [7, 8]. This may also be related to surgical manipulations including anatomical lung resections (lobectomy, pneumonectomy) and mediastinoscopy or VAMLA [7, 9-11].

Non-small cell lung cancer patients are potential candidates for surgical resection after VAMLA. If a patient with UVCP undergoes lung resection, the untreated UVCP may cause a higher risk for aspiration events and subsequent pulmonary complications such as pneumonia and atelectasis. On the other hand, oral calorie intake of these patients may be impaired due to aspiration [7, 9-11]. Lung resection surgery would cause loss of lung volume which would make the patient vulnerable to potentially life-threatening consequences related to aspiration. This makes it necessary to solve the problem urgently.

Acute injection laryngoplasty (ILP) under general anesthesia has been used for thoracic-surgery-related UVCP [7, 8]. Many authors have stated that it is a helpful technique for the immediate correction of swallowing and vocal impairment.

\section{Aim}

VAMLA-related UVCP and management with ILP has not been specifically published before. The primary objective of this study was to evaluate the effectiveness and safety of injection laryngoplasty using a temporary injectable agent (hyaluronic acid) in the acute postoperative period of patients with UVCP following VAMLA, and its positive effect on the ongoing surgical treatment of these patients.

\section{Material and methods}

Data of the patients who underwent ILP for UVCP caused by VAMLA at our institute were retrospectively evaluated. The data were gathered from a prospective database. All patients have signed informed consent for intervention and participating in the study. The study was performed in an academic medical center (Government Supported University Hospital). All of the operations were performed in a single thoracic surgery department and the patients were consulted by the Ear, Nose and Throat (ENT) department in the same hospital which has a dedicated team for ILP. Evaluation, ILP and follow-up of the data were performed together as team work where all the authors contributed equally. If the TNM staging was appropriate, the same thoracic surgery team performed anatomical lung resections, after the ILP procedure. Patients who underwent VAMLA for mediastinal evaluation between January 2012 and January 2017 and had symptoms related to UVCP were reviewed. Inclusion criteria for the

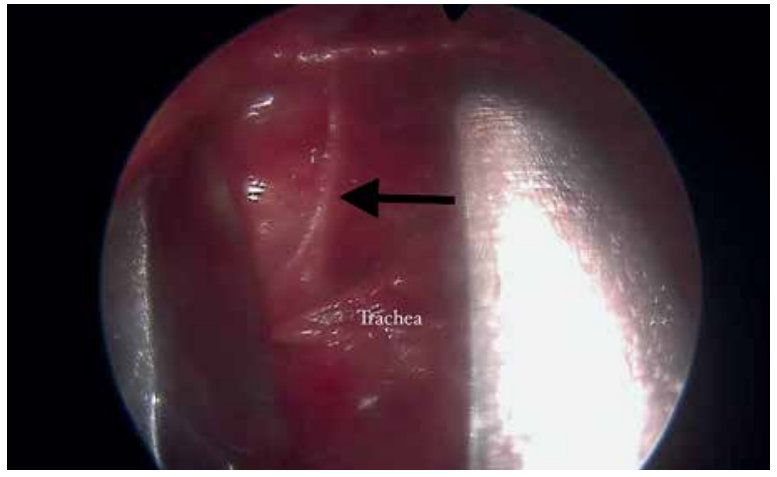

Photo 1. Left recurrent nerve (black arrow) may be visible after resection of the left lower paratracheal lymph nodes 
study were as follows: patients with a normal voice pattern before VAMLA, patients with UVCP-associated symptoms which occurred after VAMLA, patients whose diagnosis of UVCP was confirmed by video laryngoscopy by visualizing the vocal cord in the paramedian localization; glottic insufficiency during adduction which was not compensated by the other cord could be visualized; patient who was tested for aspiration of liquid and solid substances and also for the ability of effective coughing.

Exclusion criteria were as follows: patients with diagnosis of vocal cord palsy before VAMLA, patients whose UVCP was caused by other etiologies, no evidence of denervation, unable to cooperate with evaluations, patients with voice problems but having no sign of aspiration during oral intake and who can cough effectively.

In addition to detailed examination with the video laryngoscope, patients were asked to complete the Voice Handicap Index-10 (VHI-10). The Voice Handicap Index-10 is a questionnaire which measures a patient's self-perception of voice handicap $[12,13]$. The VHI-10 consists of 10 statements comprising a shortened version of the original 30 item Vocal Handicap Index. Each statement on the 10-question instrument is rated with a 0 to 4 point Likert scale, with 0 indicating "never" and 4 indicating "always" (maximum score of 40, minimum score of 0 ). The total score is used to indicate the severity of the voice disorders for the patient. Higher scores indicate that a voice problem has a more severe handicapping effect on the individual's life than a lower score [12-14]. Maximum phonation time (MPT) is also a clinical tool for assessing phonatory mechanics. MPT is defined as the longest period during which a patient can sustain phonation of a vowel sound, typically /a/. A timer and audio recorder are the only equipment typically used to measure MPT. Maximum phonation time has been used to measure laryngeal function indirectly in pathological circumstances, including patients with paralytic dysphonia [15, 16]. Maximum phonation time has been used for assessment of the severity of dysphonia and for the assessment of improvement following the therapy in patients with dysphonia [17].

Patients' aspiration status during both intake (yes-no), effective coughing and sputum expectoration (yes-no) status was evaluated and recorded. For the minimally invasive ILP procedure the patient was placed in the supine position on the operating room table. The patient was intubated by the anesthesiologist and under general anesthesia. The laryngoscope was inserted in the standard fashion, and the true vocal cords were then inspected. Using the operating microscope for precise guidance, a long, 25 -gauge injection needle was placed at the level of the tip of the vocal process of the arytenoid, with the needle being directed deep into the paraglottic space. Hyaluronic acid (total of $1 \mathrm{cc}$, divided into 3 parts) was then injected into the cord until the vocal cord was medially located, just past the midline. The needle was then removed from the vocal cord and the patient was awakened. Patients were discharged a few hours after follow-up. The medical records of the patients were reviewed regarding age, sex, side of lung tumor, side of UVCP, the status of RLN at the end of VAMLA (known or unknown for transection), type of surgical resection, postoperative complications related to ILP. Pre- and postoperative measurements were all completed within 3 days (before and after the ILP) (Table I).

\section{Statistical analysis}

All statistical tests were two-sided and $p<0.05$ was considered statistically significant. Statistical analysis of continuous variables was performed using Student's $t$-test and the analysis of variance (ANOVA) test, and the $\chi^{2}$ test was used for categorical variables. Statistical analysis was performed using SPSS 10.0 (SPSS Incorporated, Chicago, IL).

\section{Results}

There were 525 NSCLC patients who underwent VAMLA intervention for evaluation of mediastinal involvement. There were $8(1.5 \%)$ patients who had dysphonia following VAMLA. During evaluation 3 patients were found to have either partial movement of the affected cord or effective compensation by the video laryngoscopy as the other vocal cord was closing the area totally. These patients were effectively coughing and there was no sign of aspiration during oral intake, so these were excluded from the study. The remaining 5 patients were enrolled in the study. Hence the rate of UVCP requiring ILP was $0.95 \%$. Four (80\%) of the patients were male, and 1 (20\%) was female. Four (80\%) of the patients were diagnosed with NSCLC on the left side while 1 (20\%) had a tumor on the right side. All of the patients (100\%) were found to have aspiration 
symptoms during oral intake. In the videolaryngoscopic examination, all patients were found to have UVCP on the left side. Histopathological results for VAMLA revealed no mediastinal tumor metastases (NO disease), so all of the patients underwent surgical lung resection. The ILP was performed immediately after the diagnosis of UVCP was made and during the histopathological examination period, so there was no delay in the surgical schedule. Before surgery, all patients regained an effective coughing reflex and there was no sign of aspiration in any of the patients. Maximum phonation time was measured before and after ILP, and the results were $7.1 \pm 1.6$ and $11.1 \pm 2.3 \mathrm{~s}$, respectively $(p<0.001)$. The Voice Handicap Index-10 score (VHI-10) was $30.4 \pm 4.7$ and $13.4 \pm 3.5$ respectively $(p<0.001)$ (Table II). Three patients underwent left upper lobectomy, 1 patient underwent left lower lobectomy, and 1 of them underwent right upper lobectomy with chest wall resection. Postoperative lymph node dissection revealed that all the patients had pNO disease. There was no morbidity or mortality related to ILP and anatomical lung resection.

\section{Discussion}

The use of ILP in the treatment of UVCP provides a stable position for the paralytic vocal cord on the midline which helps to establish glottal function [7, $8,18]$. This improves the voice quality, but more than that, it avoids aspiration problems and reestablishes the coughing function. This prevents atelectasis and infectious situations which may cause more complex clinical situations for these patients who are lung resection candidates. Conservative treatment with 9-12 months follow-up is another option but it is not appropriate for these patients since they have a malignancy which needs an urgent intervention $[18,19]$. There are new approaches of office-based injection laryngoplasty which is performed under local anesthesia with successful outcomes [20].

VAMLA is an excellent tool for staging NSCLC and planning a correct and further treatment plan (sur-
Table I. Patient characteristics and results

\begin{tabular}{|c|c|}
\hline Parameter & Value \\
\hline Age, mean (range) [years] & $\begin{array}{c}62.8 \\
(54-72)\end{array}$ \\
\hline \multicolumn{2}{|l|}{ Gender: } \\
\hline Male & $4(80 \%)$ \\
\hline Female & $1(20 \%)$ \\
\hline \multicolumn{2}{|l|}{ Nerve damage status: } \\
\hline Not known & $5(100 \%)$ \\
\hline Transection & $0(0 \%)$ \\
\hline \multicolumn{2}{|l|}{ Tumor location: } \\
\hline Left & $3(60 \%)$ \\
\hline Right & $2(40 \%)$ \\
\hline \multicolumn{2}{|l|}{ Side of paralysis: } \\
\hline Left & $5(100 \%)$ \\
\hline Right & $0(0 \%)$ \\
\hline \multicolumn{2}{|l|}{ Aspiration with liquids before injection: } \\
\hline Yes & $5(100 \%)$ \\
\hline No & $0(0 \%)$ \\
\hline \multicolumn{2}{|l|}{ Dysphonia before injection: } \\
\hline Yes & $5(100 \%)$ \\
\hline No & $0(0 \%)$ \\
\hline \multicolumn{2}{|l|}{ Lung surgery performed: } \\
\hline Left upper lobectomy & $2(40 \%)$ \\
\hline Left lower lobectomy & $1(20 \%)$ \\
\hline $\begin{array}{l}\text { Right upper lobectomy and chest wall } \\
\text { resection }\end{array}$ & $1(20 \%)$ \\
\hline Right lower lobectomy & $1(20 \%)$ \\
\hline \multicolumn{2}{|l|}{ Post-injection problem: } \\
\hline Mortality & $0(0 \%)$ \\
\hline Morbidity & $0(0 \%)$ \\
\hline \multicolumn{2}{|c|}{ Post-resection problem related to vocal cord dysfunction: } \\
\hline Mortality & $0(0 \%)$ \\
\hline Morbidity & $0(0 \%)$ \\
\hline
\end{tabular}

Table II. Maximum phonation time (MPT) and Voice Handicap Index (VHI-10) results before and after ILP

\begin{tabular}{|lccc|}
\hline Parameter & $\begin{array}{c}\text { Mean value before injection } \\
\pm \text { standard deviation }\end{array}$ & $\begin{array}{c}\text { Mean value after injection } \\
\pm \text { standard deviation }\end{array}$ & $P$-value \\
\hline Maximum phonation time (MPT) & $7.1 \pm 1.6$ & $11.1 \pm 2.3$ & $<0.001$ \\
\hline Voice Handicap Index (VHI-10) & $30.4 \pm 4.7$ & $13.4 \pm 3.5$ & $<0.001$ \\
\hline
\end{tabular}


gery vs. non-surgery) $[1,4]$. VAMLA not only protects NSCLC patients from unnecessary surgery but improves surgical results due to correct patient selection [4]. We have been performing VAMLA in patients with the diagnosis of NSCLC who have the chance to be candidates for surgical resection after mediastinal evaluation. We perform mediastinal evaluation when computed tomography (CT) shows enlarged mediastinal nodes and when positron emission tomography (PET) shows increased uptake in the mediastinum or the hilum, and of course in central tumors as recommended by the recent guidelines. Confirmation of the mediastinal involvement with pathological examination of tissue samples provided by endoscopic techniques or with open surgical intervention is crucial for detailed staging. Moreover, preoperative mediastinal staging is advised for tumors larger than $3 \mathrm{~cm}$, especially in adenocarcinomas with a high standardized uptake value $[1,4]$.

Our prior experience of VAMLA has shown a better negative predictive value, better sensitivity, better false-negative rate, and better accuracy in patients who underwent VAMLA, compared with standard mediastinoscopy [4]. Five-year survival was $90 \%$ for the VAMLA group and $66 \%$ for the mediastinoscopy group, and there was a statistically significant difference in favor of the VAMLA group [4]. This outcome could not be explained by any mechanism, but this is a topic that we continue working on. In VAMLA technically we are performing a deep excision of the mediastinal lymph node areas. There is a risk of damaging the recurrent laryngeal nerve mostly on the left side while excising the left paratracheal lymph nodes.

In our previously published experience, dysphonia was noted in $8(9.0 \%)$ patients who underwent VAMLA, whereas the complication rate was $4.1 \%$ (13 dysphonia and 1 pneumothorax) in patients who underwent standard mediastinoscopy [4]. The rate of UVCP decreases significantly as the experience of the surgeons increases, which was lower in this patient group with a rate of $1.5 \%$, and the rate of ILP requirement was even less. For UVCP there is a possibility of adequate compensation by the contralateral vocal cord if the nerve is not transected, but these patients do not have sufficient time to wait because they have a neoplastic disease that requires urgent intervention. Thus any patient with UVCP after VAMLA should be evaluated and treated rapidly. Early onset of an intervention to improve laryngeal function in these patients is very important.
This allows early alleviation of the impact on their quality of life, including the breathy dysphonia [11]. Previous studies $[7,8]$ have reported outcomes of ILP for the treatment of UVCP following various thoracic surgery procedures, but this study seems to be the first original paper focusing on UVCP following VAMLA. The patients evaluated in our series were potential candidates for a resection which makes injection laryngoplasty and its favorable results more important in these patients. Acute treatment of VAMLA-related UVCP with injection laryngoplasty appears to be a safe and effective method for preventing postoperative aspiration pneumonia. Single injection laryngoplasty can be offered to this group of patients as a first line treatment method with satisfactory results.

\section{Conclusions}

Video-assisted mediastinoscopic lymphadenectomy has been proven to be a successful staging tool for NSCLC. However, it is not performed routinely in most thoracic surgery clinics. In our opinion, it should be used more frequently and should be popularized. The technical difficulties and potential risk for complications, mostly UVCP, may be frightening for beginners. This should not be considered as a limitation because the risk of UVCP decreases as the experience of the surgeon increases. Moreover, multidisciplinary team work and ILP seems to be a successful and effective way of managing this complication in patients who are diagnosed with UVCP.

\section{Conflict of interest}

The authors declare no conflict of interest.

\section{References}

1. Call S, Obiols C, Rami-Porta R, et al. Video-assisted mediastinoscopic lymphadenectomy for staging non-small cell lung cancer. Ann Thorac Surg 2016; 101: 1326-33.

2. Silvestri GA, Gonzalez AV, Jantz MA, et al. Methods for staging non-small cell lung cancer: diagnosis and management of lung cancer. 3rd ed: American College of Chest Physicians evidence-based clinical practice guidelines. Chest 2013; 143: 211-50.

3. Leyn PD, Dooms C, Kuzdzal J, et al. Revised ESTS guidelines for preoperative mediastinal lymph node staging for non-smallcell lung cancer. Eur J Cardiothorac Surg 2014; 45: 787-98.

4. Turna A, Demirkaya A, Ozkul S, et al. Video-assisted mediastinoscopic lymphadenectomy is associated with better survival 
than mediastinoscopy in patients with resected non-small cell lung cancer. J Thorac Cardiovasc Surg 2013; 146: 774-80.

5. Yasufuku K, Pierre A, Darling G, et al. A prospective controlled trial of endobronchial ultrasound-guided transbronchial needle aspiration compared with mediastinoscopy for mediastinal lymph node staging of lung cancer. J Thorac Cardiovasc Surg 2011; 142: 1393-400.

6. Heurtgen M, Friedel G, Toomes H, Fritz P. Radical video-assisted mediastinoscopic lymphadenectomy (VAMLA): technique and first results. Eur J Cardiothorac Surg 2002; 21: 348-51.

7. Graboyes EM, Bradley JP, Meyers BF, et al. Efficacy and safety of acute injection laryngoplasty for vocal cord paralysis following thoracic surgery. Laryngoscope 2011; 121: 2406-10.

8. Fang TJ, Hsin LJ, Chung HF, et al. Office-based intracordal hyaluronate injections improve quality of life in thoracic-surgery-related unilateral vocal fold paralysis. Medicine (Baltimore) 2015; 94: e1787.

9. Gockel I, Kneist W, Keilmann A, et al. Recurrent laryngeal nerve paralysis (RLNP) following esophagectomy for carcinoma. Eur J Surg Oncol 2005; 31: 277-81.

10. Hulscher JB, van Sandick JW, Devriese PP, et al. Vocal cord paralysis after subtotal oesophagectomy. Br J Surg 1999; 86: 1583-7.

11. Baba M, Natsugoe S, Shimada M, et al. Does hoarseness of voice from recurrent nerve paralysis after esophagectomy for carcinoma influence patient quality of life? J Am Coll Surg 1999; 188: 231-6.

12. Deary IJ, Webb A, Mackenzie K, et al. Short, self-report voice symptom scales: psychometric characteristics of the voice handicap index-10 and the vocal performance questionnaire. Otolaryngol Head Neck Surg 2004; 131: 232-5.

13. Rosen CA, Lee AS, Osborne J, et al. Development and validation of the voice handicap index-10. Laryngoscope 2004; 114: 1549-56.

14. Young VN, Jeong K, Rothenberger SD, et al. Minimal clinically important difference of voice handicap index-10 in vocal fold paralysis. Laryngoscope 2017; 8 doi: 10.1002/lary.27001. Epub ahead of print.

15. Maslan J, Leng X, Rees C, et al. Maximum phonation time in healthy older adults. J Voice 2011; 25: 709-13.

16. Arnold GE. Vocal rehabilitation of paralytic dysphonia: II. Acoustic analysis of vocal function. Arch Otolaryngol 1955; 62: 593-601.

17. Speyer R. Effects of voice therapy: a systematic review. J Voice 2008; 22: 565-80.

18. Caton T, Thibeault SL, Klemuk S, et al. Viscoelasticity of hyaluronan and nonhyaluronan based vocal fold injectables: implications for mucosal versus muscle use. Laryngoscope 2007; 117: 516-21.

19. Yung KC, Likhterov I, Courey MS. Effect of temporary vocal fold injection medialization on the rate of permanent medialization laryngoplasty in unilateral vocal fold paralysis patients. Laryngoscope 2011; 121: 2191-4.

20. Sielska-Badurek EM, Sobol M, Jędra K, et al. Injection laryngoplasty as miniinvasive office-based surgery in patients with unilateral vocal foldparalysis - voice quality outcomes. Videosurgery Miniinv 2017; 12: 277-84.

Received: 31.01.2018, accepted: 26.02.2018. 